\title{
Quasi-universal scaling in mouse-brain neuronal activity stems from edge-of-instability critical dynamics
}

\author{
Guillermo B. Morales ${ }^{\mathrm{a}}$, Serena Di Santo ${ }^{\mathrm{b}}$, Miguel A. Muñoz ${ }^{\mathrm{a}, *}$ \\ ${ }^{a}$ Departamento de Electromagnetismo y Física de la Materia and Instituto Carlos I de Física Teórica y \\ Computacional. Universidad de Granada. E-18071, Granada, Spain \\ ${ }^{b}$ Center for Theoretical Neuroscience, Mortimer B. Zuckerman Mind Brain Behavior Institute, Columbia University, \\ New York, NY USA
}

\begin{abstract}
The brain is in a state of perpetual reverberant neural activity, even in the absence of specific tasks or stimuli. Shedding light on the origin and functional significance of such activity is essential to understanding how the brain transmits, processes, and stores information. An inspiring, albeit controversial, conjecture proposes that some statistical characteristics of empirically observed neuronal activity can be understood by assuming that brain networks operate in a dynamical regime near the edge of a phase transition. Moreover, the resulting critical behavior, with its concomitant scale invariance, is assumed to carry crucial functional advantages. Here, we present a data-driven analysis based on simultaneous high-throughput recordings of the activity of thousands of individual neurons in various regions of the mouse brain. To analyze these data, we construct a unified theoretical framework that synergistically combines cutting-edge methods for the study of brain activity (such as a phenomenological renormalization group approach and techniques that infer the general dynamical state of a neural population), while designing complementary tools. This unified approach allows us to uncover strong signatures of scale invariance that is "quasi-universal" across brain regions and reveal that these areas operate, to a greater or lesser extent, at the edge of instability. Furthermore, this framework allows us to distinguish between quasi-universal background activity and non-universal input-related activity. Taken together, the following study provides strong evidence that brain networks actually operate in a critical regime which, among other functional advantages, provides them with a scale-invariant substrate of activity in which optimal input representations can be sustained.
\end{abstract}

Keywords: Criticality | Scaling | Neural dynamics | Renormalization Group | Neural representations|

\section{Introduction}

The cerebral cortex of mammals is in a state of continuous ongoing activity even in the absence of stimuli or specific tasks $[1,2,3]$. Shedding light onto the origin and functional meaning of such an energy-demanding baseline state of background dynamics and its interplay with input-evoked activity are challenging goals, essential to ultimately understand how the cortex represents, processes and transmits information $[2,1,4,5,6,7,8]$. Following the fast-paced development of powerful neuroimaging and electrophysiological technologies such as, e.g., two-photon calcium imaging [9] and neuropixels probes [10], recent years have witnessed important advances in our understanding of these issues.

An inspiring hypothesis - which aims to become a general principle of brain dynamical organizationposits that neuronal networks could achieve crucial functional advantages, including optimal information processing and transmission, by operating in the vicinity of a critical point $[11,12,13]$. Using the

\footnotetext{
*Corresponding author mamunoz@onsager.ugr.es
} 
jargon of statistical physics, this implies that the network operates in an intermediate regime at the border between "ordered" and "disordered" phases [14, 15, 16, 17, 18, 19, 20, 21, 22, 23, 24, 25, 26]. Criticality, with its concomitant power-laws, entails well-known functional advantages for information processing such as, e.g., an exquisite sensitivity to perturbations and a huge dynamic range $[16,13,27]$ to name a few. In particular, it also gives raise to the emergence of a broad spectrum of spatio-temporal scales, i.e. scale invariance or simply "scaling" [13, 28, 29], that could be essential to sustain brain activity at a broad hierarchy of scales, as required to embed highly-diverse cognitive processes.

In spite of its conceptual appeal and thrilling implications, the validity of this so-called "criticality hypothesis" as an overarching principle of dynamical brain organization remains controversial [30, 31, 32]. Therefore, novel theoretical approaches and more-stringent experimental tests are much needed to either prove or disprove this conjecture and, more in general, to advance our understanding of the mechanisms underlying brain ongoing activity.

From the theoretical side it is crucial to refine the conjecture itself and discern what type of criticality is the most pertinent to describe brain activity $[11,13]$. Different possible scenarios have been explored; among them [13]: (i) the edge of propagation of activity, with scale-free avalanching behavior [14, 15, 18, 33], (ii) the edge of a synchronization phase transition [34, 24, 35, 36, 13], and (iii) the edge of a stable-unstable transition, often called "edge of chaos" but to which we will refer as "edge of instability" hereon [37, 38, 39].

From the empirical side, evidence of putative brain criticality often relies on the detection of scalefree bursts of activity, called "neuronal avalanches" [14, 40, 22, 41, 35, 42], the presence of robust long-range spatio-temporal correlations [43, 44], the analysis of large-scale whole-brain models fitted to match empirically observed correlations [45, 46, 47, 48], etc. Recently, with the advent of modern techniques enabling simultaneous recordings of thousands of neurons, complementary experimental evidence revealing the existence of scaling in brain activity - which might or might not stem from underlying criticality $[11,13,32,49]$ — has emerged from unexpected angles. Among these, let us mention (i) a novel renormalization-group approach which identified strong signatures of scale-invariant activity in recordings of more than a thousand neurons in the mouse hippocampus [50, 51], (ii) the direct inference - using linear response theory and methods from the physics of disordered systemsof "edge-of-instability" type of critical behavior in neural recordings from the macaque monkey motor cortex [39], and (iii) the discovery of an unexpected power-law, which revealed scale invariance in the spectrum of the activity covariance matrix from tens of thousands of neurons in the mouse V1 cortex while the mouse was exposed to a large set of sequentially-presented natural images [8].

What all these works have in common, is the fact the they use specifically-designed, powerful mathematical tools to analyze vast amounts of high-throughput data. Thus, these approaches not only open up new and exciting research avenues but, even more importantly, they allow for synergistic approaches to exploit them together, paving the road to further advances.

Here we propose an unified theoretical framework and employ it to analyze state-of-the-art neural recordings in diverse areas of the mouse brain [52]. As we show in what follows, our analyses strongly enhance our understanding of scale-invariance and possible criticality in the brain. In particular, this allows us to elucidate the emergence of quasi-universal scaling across regions in the mouse brain, to conclude that all such regions are, to a greater or lesser extent, posed at the edge of instability, as well as to disentangle background and input-evoked neural activities.

\section{Theoretical framework and open-ended questions}

For the sake of self-containedness, let us briefly discuss the three innovative theoretical approaches cited above, along with some stemming open-ended questions that we pose (further technical details are deferred to the Methods section).

(A) Renormalization-group approach to neuronal activity. The renormalization group $(\mathrm{RG})$ is retained as one of the most powerful ideas in theoretical physics, allowing us to rationalize 
collective behavior - at broadly-diverse observational scales - from the properties of the underlying "microscopic" components, and to understand, for instance, the emergence of scale invariance [29, 53]. In a remarkable contribution, Meshulam et al. developed an RG approach to analyze time series from large populations of simultaneously recorded individual spiking neurons and scrutinize their collective behavior [50, 51]. The method, similar in spirit to Kadanoff's blocks for spin systems [29], allows one to construct effective descriptions of time-dependent neural activity at progressively larger "coarsegrained" scales. Notably, a number of non-trivial features - generally attributed to scale-invariant critical systems - emerge from the application of such RG analyses to recordings of more than 1000 neurons in the mouse hippocampus while it is moving in a virtual-reality environment. These features include among others: (i) a non-Gaussian (fixed-point) distribution of neural activity at large scales, (ii) non-trivial scaling of the activity variance and auto-correlation time as a function of the coarsegraining scale, and (iii) a power-law decay of the spectrum of the covariance-matrix [50, 51, 54].

A limitation of this type of RG analysis is that, even if it is capable of uncovering scale invariance, it does not allow discerning what kind of putative phase transition could be at the roots of the observed scale and, moreover, doubts have been raised about the possible interpretation of the results as stemming from criticality (see $[55,56]$ and below).

In any case, leaving aside for the time being these caveats, one can wonder whether the observed scaling features are shared by other brain regions or if they are rather specific to the mouse hippocampus. Is there any kind of "universality" in the neural dynamics across brain regions despite their considerable anatomical and functional differences? Is it possible to find empirical evidence that allows us to discern whether the observed scaling actually stems from critical behavior and is not a mere consequence of, for example, unobserved latent inputs?

(B) Inferring the dynamical regime from neural recordings. Dahmen et al. devised a general approach — based on linear-response theory ideas and tools from the physics of disordered systems - that allows one to infer the overall dynamical state of an empirically observed neuronal population. In particular, this theoretical approach permits to infer the distance to the "edge of instability" from empirical measurements of the mean and dispersion of "spike-count covariance" across pairs of recorded neurons [39]. ${ }^{1}$ Straightforward application of this approach to neural recordings from motor cortex of awake macaque monkeys strongly supports the idea that such a region operates in a "dynamically balanced critical regime with nearly unstable dynamics", that they called "secondtype criticality" (see below) [39]. Thus, one can wonder whether other brain regions - for instance, the hippocampus in (A) - can also be empirically proven to be similarly close to the edge of instability.

Moreover, recently $\mathrm{Hu}$ and Sompolinsky went a step further and derived an analytical expression for the full spectrum of eigenvalues of the spike-count covariance matrix in the limit of large network sizes. This analysis reveals that the eigenvalue distribution develops a power-law tail if (and only if) the intrinsic dynamics is at the edge of instability [58]. Crucially, such a non-trivial power-law behavior necessarily stems from the recurrent network dynamics at the edge of instability [58], as it cannot be possibly ascribed to, e.g., limited sampling [59] or hidden variables [55]. Thus, we also ask whether the eigenvalue distribution of spike-count covariance matrices, as estimated from available empirical data, do exhibit power-law tails implying critical behavior. If so, this approach could provide us with even more stringent evidence of near-critical dynamics across brain regions.

(C) Scaling in optimal input representations. Stringer et al. [8] studied theoretically and experimentally the spectrum of the covariance matrix in neuronal populations in the mouse V1 visual cortex while the mouse was exposed to a very large set of sequentially-presented natural images (note that they recorded more than $10^{4}$ neurons in parallel; see Methods). From the resulting data they found that the spectrum of the covariance matrix obeyed "an unexpected power law" [8]: the n-th rank-ordered eigenvalue scaled as $1 / n^{\mu}$ with $\mu \gtrsim 1$.

\footnotetext{
${ }^{1}$ Let us emphasize that this covariance, also called "noise covariance", is not to be confused with "signal covariance" (referred to simply as "covariance" hereon), which is the one employed in the RG approach and other analyses (see Methods and $[57,58])$.
} 
This power-law decay of the rank-ordered eigenspectrum was somehow surprising; the authors were expecting a faster decay, as would correspond to a lower-dimensional representation of the visual inputs (see below). Notice that, here, by "representations" one means neural activity that stems from or is correlated with sensory or task-related inputs [60]. On the other hand, the term "dimensionality" is employed in the sense of "principal component analysis" (PCA) [61], where the dimension is the number of principal components required to explain a given percentage of the total variance; often most of the variability in neural data can be recapitulated in just a few principal components or dimensions [62].

Remarkably, Stringer et al. were also able to prove that the power-law decay of the rank-ordered eigenvalues is not an artifact directly inherited from the statistics of the inputs, but a very deep mathematical property: it stems from a trade-off between the neural representation of visual inputs being as high-dimensional as possible (i.e. including a large number of non-negligible components in a PCA analysis) and mathematically preserving its smoothness (i.e. its continuity and differentiability). As a simple illustration of this last abstract property, let us mention that the smoothness of the representation prevents, for instance, that tiny variations in the inputs dramatically alter the neural population activity, which translates into more robust representations [8].

Let us finally recall that common knowledge in statistical physics tells us that a power-law decay of the covariance-matrix spectrum (i.e., of the "propagator") is one of the most remarkable generic trademarks of critical behavior, emphasizing the emergence of a scale-free hierarchical organization of spatio-temporal correlations $[29,63,53]$. Indeed, this spectrum is one of the objects studied in the phenomenological RG approach (A), revealing a power-law decay (for the mouse hippocampus) with an exponent $\mu<1$ [50], which disagrres with Stringer et al.'s predictions $(\mu \gtrsim 1)$.

The results by Stringer et al. trigger a cascade of questions: is the empirically-observed scaling of the spectrum of the covariance matrix a consequence of the "external" input being represented in an optimal way? In other words, does the covariance spectrum obey scaling also in the absence of inputs, i.e. for resting-state or ongoing activity? Is intrinsic criticality in the network dynamics required to "excite" such a broad spectrum of modes supporting optimal input representations? How come the exponent $\mu$ in the hippocampus is smaller than 1 in seeming contradiction with Stringer et al.'s predictions?

Thus, while (A) allows us to detect and scrutinize the presence of scaling in empirical recordings in a systematic and quantitative way, (B) provides us with practical tools to infer the actual dynamical regime of the underlying neural network, thus paving the way to ascribe empirically-reported scaling to edge-of-instability criticality, and $(\mathrm{C})$ paves the way to relate criticality to so far unexplored functional advantages for optimal input representation.

In what follows, we use these methods in a synergetic way and develop novel and complementary tools, to create a unified theoretical framework which allows us to provide data-driven answers to most of the previously raised questions and scrutinize scale invariance and criticality across regions in the mouse brain.

\section{Results}

We analyze the empirical electrophysiological data presented by Steinmetz et al. in [52], where the activity, $x(t)$, of thousands of individuals neurons (in particular, the precise times of their spikes) is simultaneously recorded at a high $200 \mathrm{~Hz}$ resolution in several mouse brain regions (as illustrated in Fig.1) These recordings include periods in which the mouse is performing some specific task and some in which is at a "resting-state". Therefore, we first separate both types of time series and analyze the corresponding "resting-state" (to which we also refer as "background" or "ongoing") activity and "task-related" activity independently, paying special attention to the former. In addition, we also consider data from recordings of the V1 visual cortex from Stringer et al. [8] (see Methods). In all 

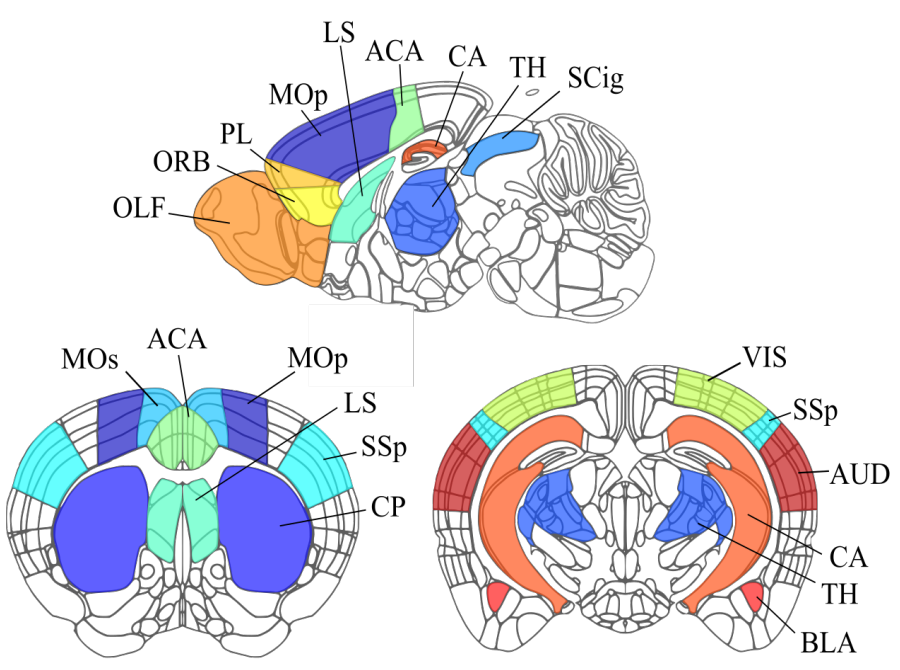

- Primary motor area (MOp)

$\checkmark$ Caudoputamen $(\mathrm{CP})$

- Thalamus (TH)

- Superior Culliculus (SCig)

* Secondary motor area (MOs)

* Primary somatosensoty area (SSp)

$\checkmark$ Lateral septal nucleus (LS)

Anterior cingulate area (ACA)

Posteromedial visual area (VISpm)

Orbital area (ORB)

Prelimbic area (PL)

Olfactory areas (OLF)

$\Delta$ Cornu ammonis (CA)

* Basolateral amygdalar nucleus (BLA)

$\star$ Auditive cortex (AUD)

Figure 1: Schematic representation of the regions in the mouse brain — using three different projections - considered in this work, together with their names and corresponding acronyms. Credit: Allen Institute, Atlas brain maps: https://atlas.brain-map.org/atlas.

cases, we restrict our analyses to areas (see Fig.1) with at least $N=256$ simultaneously-recorded neurons to ensure that scaling, if any, could be potentially observed for at least two decades.

\subsection{Quasi-universal scaling across brain regions}

We first employ the phenomenological RG approach to scrutinize whether non-trivial scaling behavior, such as the one reported in $[50,51]$ for the mouse hippocampus, is observed in other areas of the mouse brain. For the sake of clarity let us summarize the gist of the RG approach (further details in the Methods Section as well as in $[50,51])$, along with our main results.

Following the spirit of Kadanoff's block one seeks to perform a coarse graining of $N$ "microscopic variables" - i.e. single-neuron activities in this case - to construct effective descriptions at progressively larger scales. Nevertheless, given the absence of detailed information about physical connections (synapses) between neurons, a criterion of maximal pairwise correlation (rather than the standard one of maximal proximity) is used to block together pairs of neurons in a sequential way [50]. Let us remark that, for this, it is crucial to determine pairwise correlations in a careful and consistent way; this requires the choice of a suitable discrete time bin for each data set, for which we have devised an improved protocol (see Methods). In this way, the activity time series of the two most correlated neurons are added together and properly normalized, giving raise to effective time series for "blockneurons" or simply "clusters" of size 2. One then proceeds with the second most-correlated pair of neurons and so on, until all neurons have been grouped in pairs. The process is then iterated in a recursive way, so that after $k$ coarse-graining (RG) steps there remain only $N_{k}=N / 2^{k}$ block-neurons, each recapitulating the activity of $K=2^{k}$ individual neurons.

The distributions of activity values across block-neurons at level $k, P_{k}(\{x\})$, can be then directly computed for the different steps of the RG clustering procedure and, from them, a number of nontrivial features can be identified for all the considered mouse brain areas [52]:

Non-Gaussian probability distribution of block-neuron activity. Fig.2A shows the probability distribution $Q_{k}(x)$ of non-zero activity values, $x$, of the coarse-grained block-neurons at one of the RG steps $(k=5$, i.e., $K=32)$. Observe also, in the inset of Fig.2A, the excellent curve collapse obtained for sufficiently large values of $K$ as well as the presence of significant non-Gaussian tails, as exemplified for one of the considered brain regions (i.e. the primary motor cortex). This convergence of $Q_{k}(x)$ to 


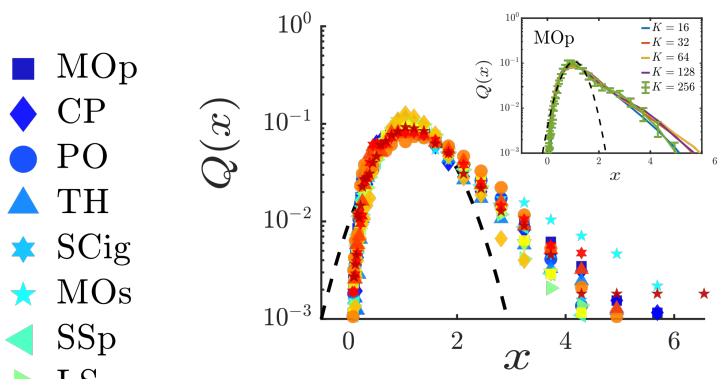

LS

ACA

VISpm

ORB

$\mathrm{PL}$

$\triangle \mathrm{CA}$

* BLA

$\star \mathrm{AUD}$
A

B

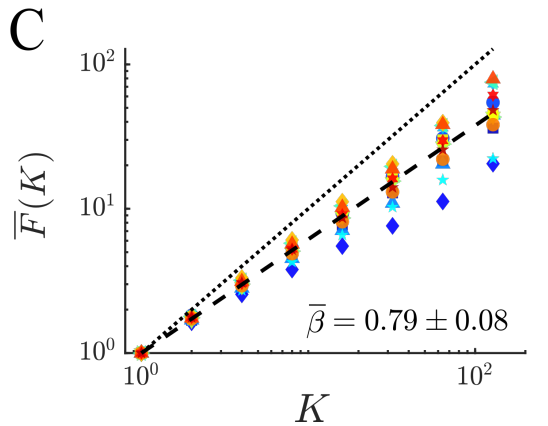

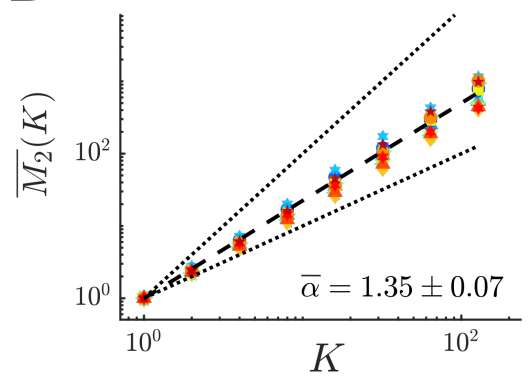

$\mathrm{D}$

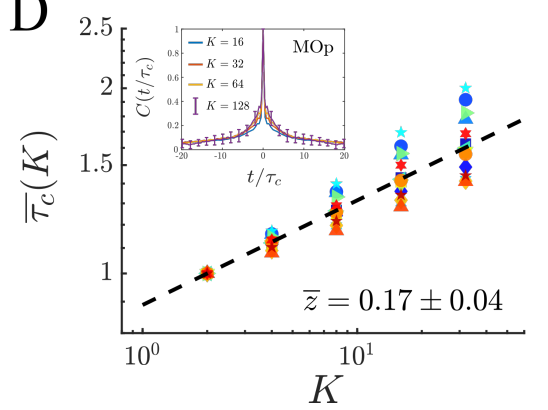

E
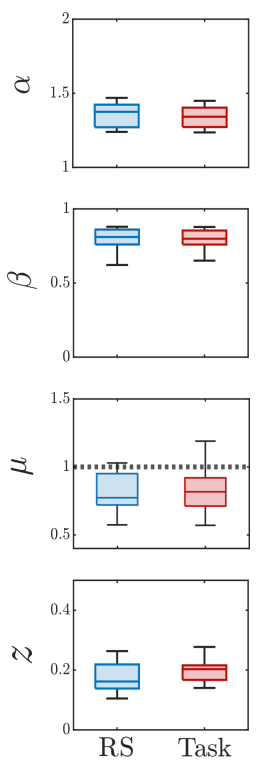

Figure 2: Results of the phenomenological RG analyses of brain activity measured in 15 different mouse brain areas (AD resting state activity). (A) Probability distribution for normalized non-zero activity in block-neurons of size $K=32$ across brain regions (main panel), as well as (inset) at 5 consecutive steps of the coarse-graining for a representative region (MOp). (B) Variance of the non-normalized activity as a function of the block-neuron size, $K$, in double logarithmic scale (upper and bottom dotted lines, with slopes 2 and 1, mark the fully correlated and independent limit cases, respectively). (C) Scaling of the free energy $F_{k}$ as defined in Eq.8 (the dotted line corresponds to the expected behavior for un-correlated variables). (D) Inset illustrates the decay of the autocorrelation function as a function of the rescaled time $t / \tau_{c}$ for the MOp region; a different value of $\tau_{c}$ is used for each cluster size but, after rescaling, all data collapse into a common curve. Main plot illustrates the scaling of the characteristic correlation time $\tau_{c}$ as a function of $K$ in double logarithmic scale for the different areas. To facilitate the comparison between areas with different number of neurons, the variance has been normalized as $\overline{M_{2}}(K)=M_{2}(K) / M_{2}(K=1)$, the probability of being silent re-scaled to $\bar{F}(K)=F(K) / F(K=1)$ and the correlation time as $\overline{\tau_{c}}(K)=\tau_{c}(K) / \tau_{c}(K=2)$. (E) Comparison of the exponent values $\alpha, \beta, \mu$ and $z$ for resting-state (RS) and task-related activity (Task). Horizontal line inside each box represents the sample median across regions, the whiskers reach the non-outlier maximum and minimum values, and the distance between the top (upper quartile) and bottom (lower quartile) edges of each box is the inter-quartile range (IQR). For the exponent $\mu$, the critical value $\mu^{c}=1$ has been marked with a dotted line. 
an asymptotic shape after a few RG steps, as observed for all regions (see SI Fig.S1), suggests that a nontrivial fixed point of the RG flow has been reached and that the emerging distribution of activity is rather universal across brain regions in resting conditions.

Scaling of the activity variance $M_{2}(K)$. As shown in Fig.2B an almost perfect scaling is observed for the variance of the non-normalized activity of block neurons, with an average exponent $\bar{\alpha}=1.35 \pm 0.07$ across areas. In particular, we measure $\alpha_{C A}=1.27 \pm 0.04$ for the CA region within the hippocampus, which can be compared with the value $\alpha=1.40 \pm 0.06$ given in [51] for such an area. Notice that all these exponent values are always in between the expected ones for uncorrelated $(\alpha=1)$ and fully-correlated variables $(\alpha=2)$, revealing consistently the existence of non-trivial scale-invariant correlations.

Scaling of the "free-energy". This is defined as $F(K)=-\log \left(S_{K}\right)$ where $S_{K}$ is the probability for a block-neuron at step $k$ to be silent (i.e. $x=0$ ) within a time bin. As shown in Fig. $2 \mathrm{C}$, this quantity exhibits a clear scaling with cluster size, with an average exponent $\bar{\beta}=0.79 \pm 0.08$ across regions $(0.88 \pm 0.03$ for the $\mathrm{CA}$ in the hippocampus, almost identical to the value $0.87 \pm 0.03$ reported in $[50])$.

Scaling of the auto-correlation time $\tau_{c}(K)$ within block-neurons. Fig.2D shows that, despite the very broad variability of intrinsic timescales for individual neurons within each region (see SI Fig.S3), dynamical scaling can be observed in the decay of the block-neuron autocorrelation times (see Methods) in all regions, with an average exponent across regions $\bar{z}=0.17 \pm 0.04$ (with $z_{C A}=0.12 \pm 0.02$ for the CA region in the hippocampus, again in perfect agreement with the one reported in [50] for this region $(0.11 \pm 0.01)$ and compatible also with the exponent values reported in [49]). As an additional test for dynamical scaling, we show in the SI (Fig.S2) how the curves for the auto-correlation functions at different coarse-grain levels collapse when time is appropriately re-scaled.

Scaling of the covariance-matrix spectrum. By diagonalizing the covariance matrix computed at different levels of the coarse-graining, it is possible to analyze how their corresponding spectra decay with the rank of the eigenvalues and how their cut-offs change with cluster size. As illustrated in Fig.(3), in all the analyzed brain areas there is a clear power-law scaling of the eigenvalues with the rank, with an average exponent across regions $\bar{\mu}=0.84 \pm 0.14$, as well as a common dependence with the fractional rank $(\mathrm{rank} / K)$, the latter manifested in the collapse of the curves at different levels of coarse graining, much as in [50]. Likewise, the value reported in [50] for the CA region in the hippocampus $(\mu=0.71 \pm 0.15)$ is in perfect agreement with our measured value for the same region: $\mu_{C A}=0.73 \pm 0.07$. Although we will address this point later on, let us for now stress that $\mu$ is smaller or, at most, approximately equal to one 1 in all regions.

For the sake of consistency, we have also verified that the reported exponent values exhibit little variability upon changes in the time-discretization bin (see Fig.S4 in SI).

Moreover, as it turns out, similar signatures of scale-invariance to those reported for restingstate activity emerge in RG analyses of neural recordings obtained while the mice are performing a visual discrimination task (see Methods and [52] for more details). This similarity is illustrated in Fig.2E, which shows how the dispersion and mean value of the scaling exponents across regions is not significantly altered when one compares the resting-state and task-related activity (for more details see Fig.S5 in the SI).

Thus, summing up, application of the phenomenological RG approach to neural recordings allows us to uncover strong empirical hints of quasi-universal scaling across regions in the mouse brain, both during resting conditions and while performing a certain task. This robust, quasi-universal behavior is striking, all the most given the limited size of the data sets and the strong region-to-region variability in intrinsic time scales and neuronal heterogeneity (see SI, Fig.S3). In any case, some differences in the quality of the scaling collapses as well as in exponent values exist between specific areas. Understanding these differences in scaling across areas and shedding light on their specificities remains an open challenge for future research. 
bioRxiv preprint doi: https://doi.org/10.1101/2021.11.23.469734; this version posted November 23, 2021. The copyright holder for this preprint (which was not certified by peer review) is the author/funder, who has granted bioRxiv a license to display the preprint in perpetuity. It is made available under aCC-BY-NC 4.0 International license.
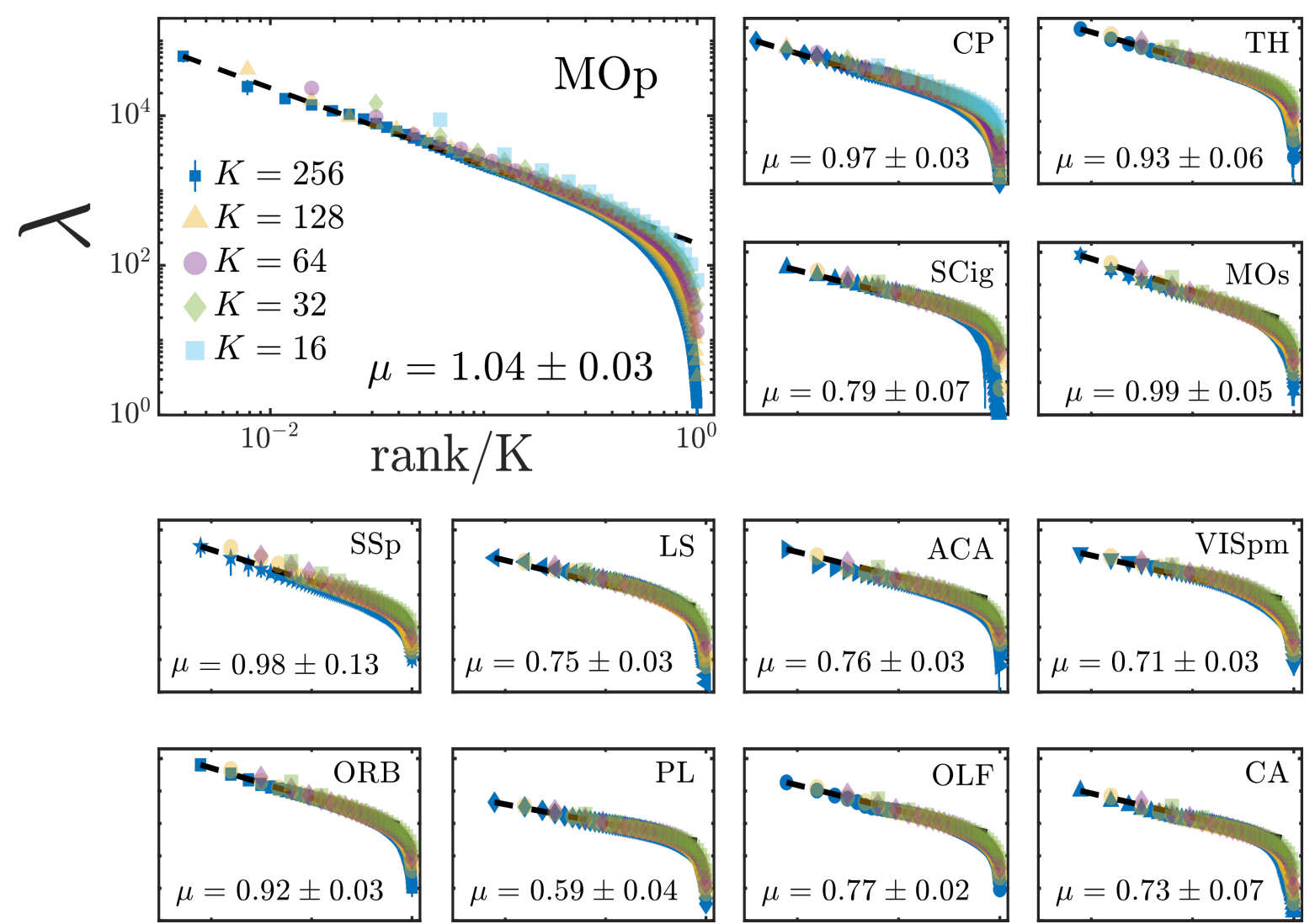

Figure 3: Scaling of the covariance matrix spectrum for the resting-state activity in clusters of size $K$, at successive steps of the RG procedure and in 13 different brain regions. Observe not only the decay of the rank-ordered eigenvalues as a power law of the rank, but also the excellent collapse of the cut-offs obtained after rescaling the eigenvalue rank by the total size $K$. For each region, errorbars in the decay exponent $\mu$ (only shown for clusters of size $K=256$ ) correspond to the standard deviation from the mean computed across random quarters of the data (see Methods). 


\subsection{Dynamical state of background activity across brain regions}

Despite the elegance and appeal of the RG results - as originally presented by Meshulam et al. - their straightforward interpretation as stemming from underlying criticality has been questioned [55, 56]. In particular, very similar scaling behavior was found to emerge in a (non-critical) model of uncoupled neurons exposed to latent correlated inputs [55] (see Discussion). Thus, it is not guaranteed a priori that the scaling behavior we just found across brain regions stems from underlying criticality and the RG approach does not allow us to provide an answer to this question. Therefore, we resort to alternative methods to estimate the dynamical regime of each brain region from empirical data. For this, one needs to compute spike-count covariance values across pairs of neurons as described above $[39,58]$. Such spike-count covariances measure the pairwise correlations in time-integrated activity (i.e. the total number of spikes) across trials/experiments, thus revealing intrinsic features of the underlying network (see Methods).

Estimations from spike-count-covariance distributions. Calling the mean value of the distribution of such spike-count covariances $\bar{c}$ and its standard deviation $\delta c$, the largest eigenvalue of the connectivity matrix, $\lambda_{\max }$ - whose closeness to 1 marks the distance to the edge of instability an thus determines the dynamical regime - can be determined from the following expression:

$$
\lambda_{\max }=\sqrt{1-\sqrt{\frac{1}{1+N \Delta^{2}}}},
$$

where $N$ is the total number of neurons in the population and $\Delta=(\delta c) / \bar{c}$ [39]. The derivation of Eq.(1) is based on linear response theory as well as tools from spin-glass theory applied to networks of spiking neurons $[64,39]$. In particular, it assumes that neural activity is stationary, a condition that typically holds during recordings of background, resting-state type of activity, but not as much in task-related activity, for which network activity is inherently input-driven and non-stationary (see SI), so we restrict our forthcoming analyses to resting-state data.

To estimate the distance to the edge of instability, $\lambda_{\max }^{c}=1$, for each brain region we considered periods of recorded resting-state activity from experimental data and split them into intervals of $10 \mathrm{~s}$, resulting in at least $N_{T}=100$ trials over which pairwise spike-count covariances were computed (see Fig.4).

As illustrated in Fig.4B (for one of the selected regions), the resulting distributions turn out to be sharply-peaked around 0, but exhibit significant tails revealing the presence of heterogeneously correlated pairs. Direct application of Eq.1 for the 15 considered regions in the mouse brain leads to the results for the maximum eigenvalue in each case, as summarized in Fig.4A (the corresponding values are collected in Table S1, in the SI). Notice that all of them lie on a relatively narrow window betwwen 0.87 and 0.96 , with a mean value $\bar{\lambda}_{\max }=0.92 \pm 0.03$, close to the edge of instability, although there is some significant region-to-region variability, with the olfatory (OLF) and prelimbic (PL) areas being the less critical ones and the thalamus (TH) the closest to the edge-of-instability. A clear understanding of these findings is still missing.

In any case, let us stress that the real dynamical state of all regions is actually closer to criticality than what is suggested by Fig.4A: indeed, it follows from Eq.1 that the value of $\lambda_{\max }$ strongly depends on the number of neurons in the region and, since the available empirical data heavily sub-samples each region, the previous approach actually underestimates the real value of $\lambda_{\max }$, which becomes much closer to 1 in the limit of tens of thousands of neurons. Thus, as shown in Fig.4C, all the analyzed regions become much closer to the edge of instability once the previous approach is extrapolated to sufficiently large, more realistic, neural population sizes (of the order of $10^{4}$ neurons).

Estimations from the eigenvalue spectrum: In a parallel analysis, we estimated the full distribution of eigenvalues of the corresponding spike-count covariance matrices, $P\left(\lambda_{\operatorname{cov}}\right)$, for each of the considered brain regions using the same data sets. From the theoretical analyses one expects to find power-law tails in such distributions if the underlying networks operate in a dynamical regime near the edge of instability [58]. For example, for random networks with Gaussian-distributed weights the theory 
A

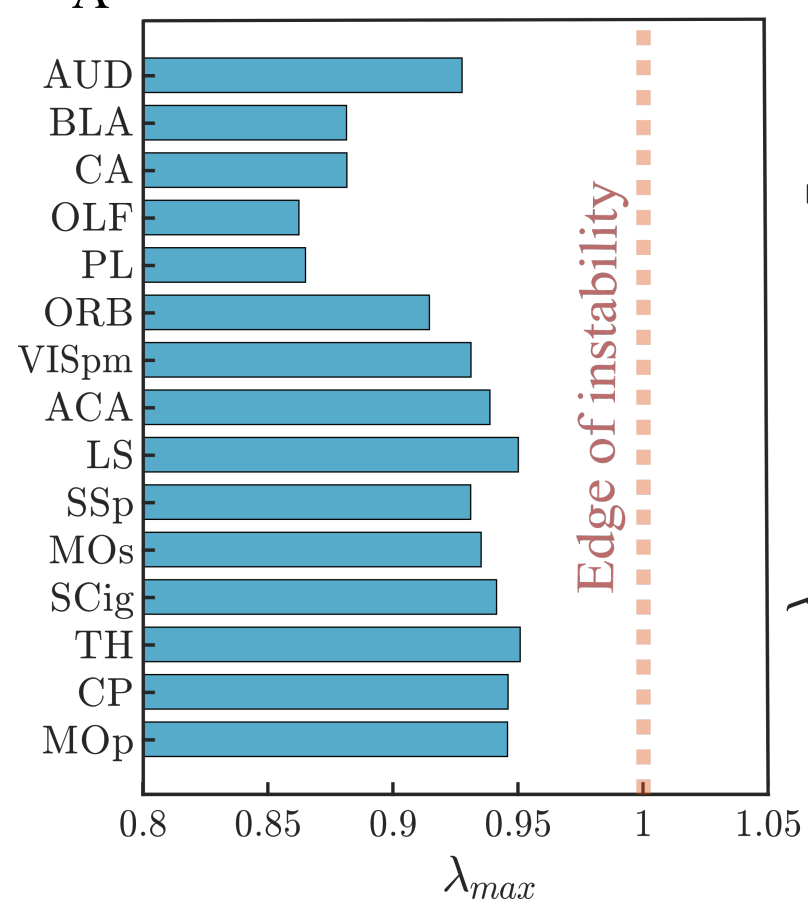

$\mathrm{B}$

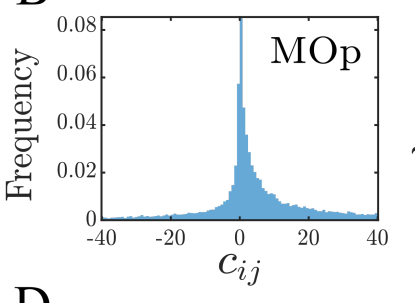

$\mathrm{D}$

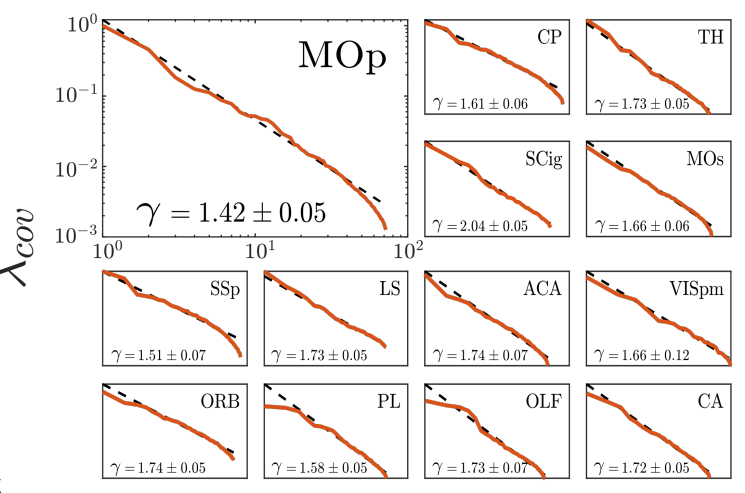

rank
C

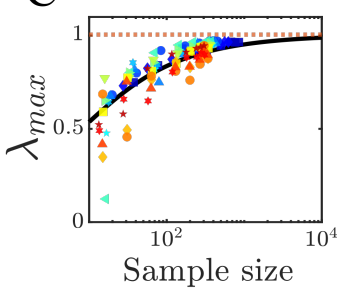

Figure 4: (A) Proximity to criticality in 15 different regions of the mouse brain, as measured by the estimated largest eigenvalue $\lambda_{\max }$ — with $\lambda_{\max }=1$ marking the "edge of instability" — of the inferred connectivity matrix; the reported values (blue bars) are lower bounds to the actual maximal eigenvalues [39]. (B) Distribution of pairwise covariance values in one of the considered brain regions (MOp). Observe the strong peak around 0 (as expected from theoretical approaches of "balanced networks" $[65,66]$ ) as well as the large dispersion of values $[39,58]$, including (asymmetric) broad tails. (C) Dependence of the estimated value of $\lambda_{\max }$ on the number of neurons $N$ in the sample. Each marker corresponds to a region as specified in Fig.1; for each region we show 10 points, corresponding to sub-samples of $10 \%$ to $100 \%$ of the neurons in the original dataset. Fitting the resulting values of $\lambda_{\max }$ for different values of $N$ to Eq.1 with a parameter $\Delta$ common to all regions and extrapolating for large values of $N$, one observes a fast convergence to the very edge of instability. (D) Rank-ordered eigenvalues of the spike-count covariance matrix for 13 of the analyzed areas. In all cases, the decay is well described as a power law for at least two decades, with exponent values as reported in the legends, not far from the theoretical prediction (for the case of Gaussian-distributed weights) in networks operating at the edge of instability [58]. To facilitate the comparison among regions, for each of them the curve has been normalized by the maximum eigenvalue of the covariance matrix. 
predicts a power-law decay of $P\left(\lambda_{\text {cov }}\right)$ with an exponent $\nu=5 / 3$ [58] or, equivalently, an exponent $\gamma=8 / 5$, with $\nu=1 /(\gamma-1)$, for the decay of its rank-ordered eigenvalues (see SI and [67] for the derivation of this scaling relation). Let us emphasize that, while the power-law behavior is generic for critical networks, the precise exponent value depends on the underlying network features, such as sparseness, excitation-inhibition balance, and weight distribution [58].

The empirically-estimated rank-ordered eigenvalues are shown in Fig.4D for some of the different brain areas. Observe that all of them can be well fitted to power-laws for about two decades; nevertheless there is some dispersion in the quality of the fits as well as in exponent values, with an average value $\bar{\gamma}=1.68 \pm 0.15$, not far from the expected result $\gamma=8 / 5$ for critical networks with Gaussian distributed weights [58]. For the sake of completeness, results for the corresponding eigenvalue probability distributions are also shown in the SI; they also exhibit clear power-law tails, with exponents $\nu$ compatible with the predictions in [58] for networks at the edge-of-instability.

Thus, summing up, we have found robust empirical evidence that all the analyzed regions in the mouse brain operate - to a greater or a lesser extent - in a dynamical regime close to the edge of instability, even if some dispersion in distances and exponent values exists across regions. Understanding these differences and their functional meaning remain as challenging goals for future work.

\subsection{Non-universal, input-dependent exponents in task-related activity}

Saying that external stimuli shape neural correlations in information processing areas is, to a certain extent, an obvious statement. However, the fact that the spectrum of the activity covariance matrix follows a very simple mathematical rule that ultimately ensures the smoothness of the internal representation of inputs — as mathematically proved by Stringer et al. [8] — is quite remarkable.

In particular, these authors showed that the neural representation for a $d$-dimensional (visual) input - that is to be encoded collectively in the neural activity of the primary visual cortex (V1) - is constrained by the requirement of smoothness of the representation (i.e., continuity and differentiability of the associated representation manifold) [8].

Being more specific, for these conditions to hold, the spectrum of the covariance matrix needs to decay as a power-law with an exponent $\mu$ that must be greater than 1 for continuity, and greater than $1+2 / d$ - where $d$ is the dimension of the input ensemble (see Methods) - for differentiability [8]. Thus, for sufficiently "complex" inputs, i.e. with large embedding dimensionality, this exponent is constrained to take a value arbitrarily close to (but larger than) unity, $\mu \gtrsim 1$.

On the other hand, in the previous RG analyses, we found values of $\mu$ consistently smaller than 1 both under resting conditions and in task-related activity - for all the considered areas (see Fig.3), in seeming contradiction with the predictions of Stringer et al. [8]. How come that we report values $\mu<1$ in all areas including sensory information encoding ones, even when the mouse is exposed to external stimuli? Does it mean that stimuli encoding violates the requirements for efficient representation put forward by Stringer et al. [8]?

At the core of this seeming paradox lies a data-processing method proposed by Stringer $e t$ al. that allows one to extract the input-only related covariances from the overall "raw" covariance matrix [8]. The approach stems from the idea that population activity can be decomposed into an input-related (or "input-encoding") subspace, which spans input-only related activity and a complementary space - orthogonal to the former one- which captures the remaining activity [60]. In a nutshell, the socalled cross-validated PCA (cv-PCA) method consists in repeating twice the very same experiment and comparing the two resulting raw covariance matrices; this comparison allows one to infer which part of the covariance is shared by the two matrices and is, hence, input-related (see Methods section) and which complementary part stems from unrelated background activity [8].

To prove that our RG results above (consistent with $\mu<1$ ) are not in blatant contradiction with the ones by Stringer et al. $(\mu \geq 1)$, we extended the cv-PCA method (as explained in detail in SI) to be able to actually extract from empirical data in [8] not just the input-related covariance matrix but also the time-series of input-related neural activity. For this, the overall activity $x(t)$ of a given 


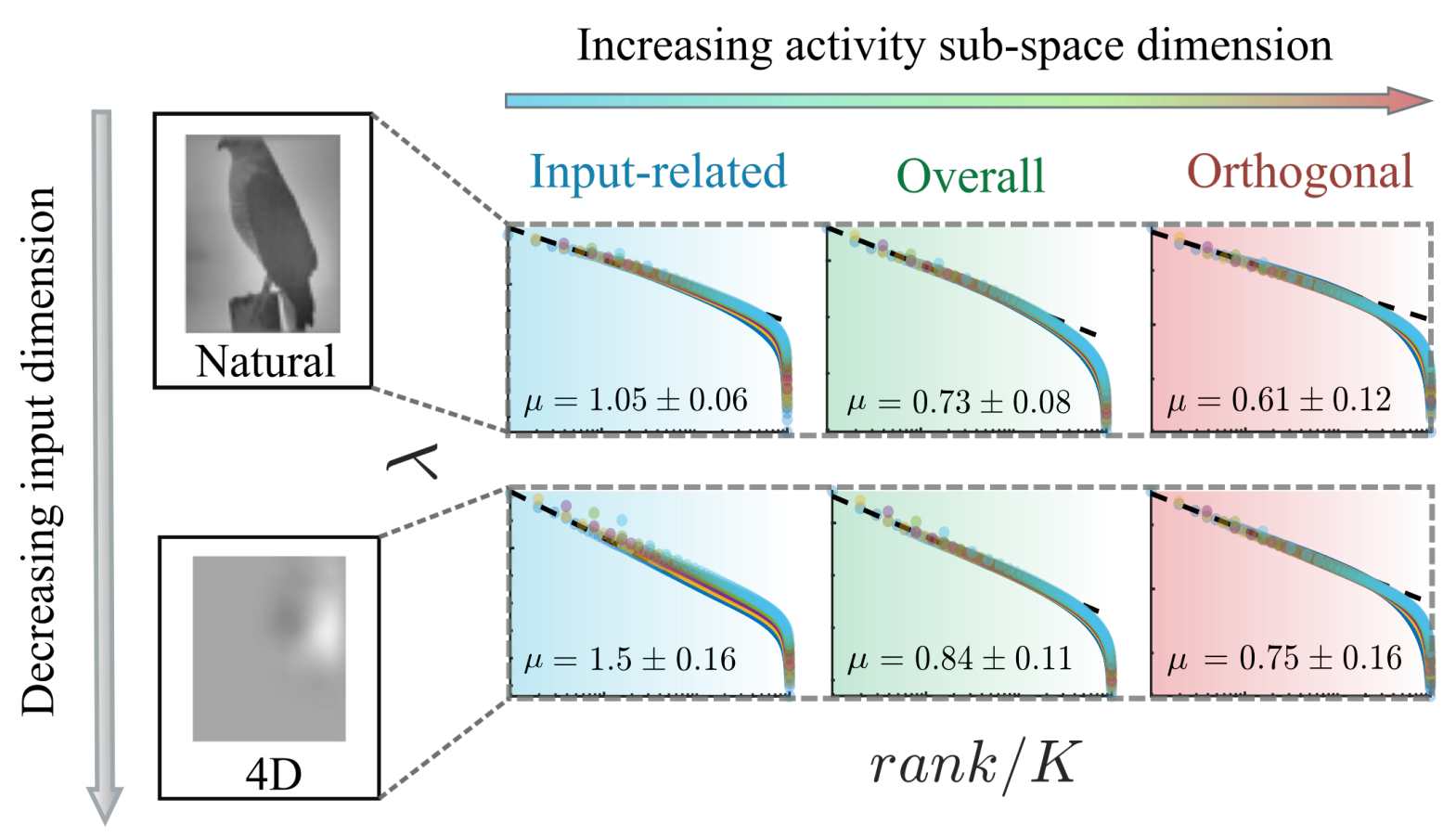

Figure 5: Diagram showing the two observed trends in the power-law exponent $\mu$, which characterizes the decay of the covariance-matrix eigenvalues as a function of their rank. $\mu$ decreases with the complexity of the input when activity is projected into the task-encoding subspace (also called representation manifold). On the other hand, for a fixed type of input, the exponent decreases with the proportion of background, "noisy" activity in the data, which lies in a higherdimensional subspace orthogonal to the representational manifold. Natural and low-dimensional images examples have been adapted from $[8]$.

neuron at time $t$ is projected into two separate sub-spaces, i.e. decomposed as:

$$
x(t)=\psi(t)+\epsilon(t)
$$

where $\psi(t)$ describes its input-related activity and $\epsilon(t)$ stands for the remaining "orthogonal" activity, responsible for trial-to-trial variability (see SI). This decomposition allows us to perform separate RG analyses to input-related and background activity data. As a consistency check, we also verified that the covariance matrix eigenspectrum associated with $\psi(t)$ has no significant difference with the one obtained from the standard application of cv-PCA analyses in [8] (see SI for further details).

We now proceed to show the main results of applying the phenomenological RG approach over (i) the raw data $x(t)$, (ii) the input-related activity $\psi(t)$ and (iii) the background activity $\epsilon(t)$, with the results averaged over three different mice.

(i) Analyzing the raw data (i.e. $x^{\prime} s$ variables) one observes again exponent values $(\alpha=1.47 \pm 0.04$ and $\mu=0.66 \pm 0.01)$ in agreement with the previously-reported quasi-universal values for different mouse-brain areas (see Fig.5 and Fig.S6 in the SI).

(ii) Considering the input-evoked activity $\left(\psi^{\prime} s\right)$ one finds that the exponents $\alpha$-which was rather robust when measured from the raw-activity data - is significantly altered (see SI, Fig.S6). More importantly, a significant increase in the exponent $\mu$ is observed with respect to the raw-data analyses; it now respects the theoretical boundary $\mu>1+2 / d$ (with $d \longrightarrow \infty$ in natural images) for the smoothness of the representation manifold (see Fig.5). In addition, the value of $\mu$ obtained from our RG analyses decreases as the input dimensionality grows, in agreement with the theoretical results and empirical findings in [8].

(iii) Finally, considering only the residual "orthogonal" parts $\left(\epsilon(t)^{\prime} s\right)$ we found slightly smaller values of $\mu$ with respect to the raw data analyses for the two type of inputs considered (see Fig.5). 
We notice, however, that this background activity is not to be confused with simple "white noise" as it exhibits correlations with a non-trivial power-law spectrum. When looking at the exponent $\alpha$ for the variance inside block-neurons, we did not observe a significant change with respect to the overall-activity case (Fig.S6 in SI). This further supports the idea that the observed scaling exponents in the overall raw data are dominated by the higher-dimensional, background activity.

To summarize the results shown in Fig.5 we recap the two observed trends in the scaling exponent $\mu$. On the one hand - for data obtained using images of different intrinsic dimensionalities (see Methods) - we found the relationship predicted and observed by Stringer et al. in [8], namely, that for the input-related activity $\psi(t)^{\prime} s$, the value of $\mu$ decreases with the complexity of the input signal, with a lower limit of $\mu=1$ for high-dimensional inputs. On the other hand, the exponent $\mu$ decreases with the relative weight of background activity in the data: the more input-related activity that is projected away, the more "noisy" (flatter) the spectrum of the remaining orthogonal space.

As a side note, let us mention that - owing to the nature of the experimental setup, in which stimuli are interspersed with grey-screen inter-stimulus intervals - computation of actual dynamical correlations are not meaningful in this case, so that we purposely left aside the study of the dynamical scaling exponent $z$. Likewise, the transformations carried on the overall data to extract the inputrelated and orthogonal activity do not necessarily preserve the biological significance of the zeroactivity, making pointless the computation of the "free-energy" exponent $\beta$.

Thus, in summary, application of the phenomenological RG procedure to the data for the V1 visual cortex in [8] reveals that the overall activity exhibits clear signatures of scale invariance, sharing its quasi-universality with the previously analyzed regions, which relied on a different dataset. Nevertheless, this overall activity can be decomposed into input-related and noisy/orthogonal activities: the scaling exponent for the covariance matrix in the first case obeys the mathematical constraints derived by Stringer et al., thus solving the seeming contradiction with the spectrum of the overall data.

\section{Discussion}

Understanding how the brain copes with inputs from changing external environments, and how information from such inputs is transmitted, integrated, processed, and stored in a physical substrate consisting of noisy neurons - exposed also to a stream of other overlapping inputs - is one of the major challenges in neuroscience. The fast development of revolutionary experimental and observational techniques as well as of novel theoretical insights have resulted in huge advances in this field in recent years.

On the one hand, novel technologies allowing for the simultaneous recording of thousands of neurons, pave the way to quantitative analyses of brain activity with unprecedented levels of resolution and detail, making it possible, for the first time, to discriminate between different overarching theories.

Here, we have taken advantage of high-throughput data, together with state-of-the-art theoretical approaches to analyze neuronal activity across regions in the mouse brain. One of our chief objectives was to make progress in elucidating whether the so-called "criticality hypothesis" — in some of its possible formulations - is supported by empirical data and, thus, can be further pursued as an overarching principle to rationalize how the intrinsic dynamics of neural networks in the brain sustains information processing. This ambitious goal has been tackled in two steps.

The first step was to assess the presence or absence of scale invariance or "scaling" in neural data, for which we extensively rely on the phenomenological RG approach recently proposed by Meshulam et al. [50, 51, 54]: our analyses here confirm the existence of strong signatures of scaling in all the analyzed brain regions, with exponent values taking quasi-universal values with only relatively small variations across areas [52]. The level of universality is not as precise as in critical phenomena in "non-living matter"; actually, roughly speaking, the exponent values change from region to region in about a ten per cent of their corresponding median value (see Fig.2). Further work is needed to better understand the origin and functional meaning of this variability.

The second step was to scrutinize whether the empirically observed scale invariance stems from 
criticality or not. Thus, the next sections are devoted to the discussion of this hypothesis at the light of our presented results.

\subsection{Criticality versus latent dynamical variables}

Importantly, as already mentioned, recent works [56, 55] have shed doubts on the possible relation between the empirically-found scaling in neural recordings and actual criticality, as originally suggested by Meshulam et al. [50, 51]. In particular, Morrell et al. constructed a very simple model of uncoupled binary neurons, which cannot possibly exhibit collective behavior such as phase transitions or criticality [55]. In their setup, all individual neurons are exposed to a large set of shared external, time-correlated inputs, so-called "latent dynamical variables" or simply "hidden variables". Surprisingly enough, such a toy model is able to reproduce - for a relatively broad region of the parameter space - the RG scaling with non-Gaussian activity distributions as well as a set of exponents roughly compatible with that in actual neural networks $[50,51]$. This suggests that the empirically observed scale invariance in neural recordings could possibly have nothing to do with intrinsic reverberating critical dynamics, but just emerge as an evoked response to shared external drivings.

Let us recall that this type of dichotomy for the interpretation of scaling — between critical behavior on the one hand and a superposition of the simpler effects stemming from hidden variables on the other - is a common theme in different branches of science, being for example at the root of discussions about the meaning of the Zipf's law in neural data [28, 68, 69, 32, 70].

In the present case, our analyses contribute to discriminate between these two possible interpretations for mouse-brain neuronal recordings. As a matter of fact, by employing a variety of tools, we have concluded that all the empirically analyzed brain regions lie - to a greater or lesser extent - at the edge of instability, i.e. in the vicinity of a critical point separating stable from linearly unstable phases.

Furthermore, as already emphasized by $\mathrm{Hu}$ and Sompolinsky, a long tail in the distribution of the eigenvalues of the spike-count covariance matrix - which is one of the chief properties we find in the analyzed data - is a distinct feature of correlations arising from nearly-unstable recurrent network dynamics [58]: it cannot be possibly reproduced by a network of uncoupled neurons and, thus, it cannot be merely an effect stemming from superposed hidden inputs.

Nevertheless, let us caution that this does not imply that latent dynamical inputs do not have an impact in the observed scaling exponents, as some differences in exponent values across regions exist, which could stem from different exposures to a constant stream of inputs from other areas. Further empirical and theoretical studies would be required to advance in this direction; in particular, developing more elaborated models including both near-critical recurrent dynamics and shared latent variables, as well as analyzing how the relative weights of both contributions shape scaling exponents remain open goals for future research.

\subsection{Edge of instability and optimal representations}

By applying the phenomenological RG procedure to the data for the V1 cortex in [8] we found that the overall activity exhibits clear signatures of scale invariance and shares its quasi-universality with the previously analyzed regions relying on a different dataset, solving a seeming contradiction between our own results for quasi-universal scaling across brain regions and those in [8]. However, the projection of the overall or "raw" activity into input-representing and complementary/orthogonal-space activities allowed us to conclude that the scaling exponent determined from RG analyses in the first case obeys the mathematical constraints derived by Stringer et al.

Finally, let us emphasize that, given that all the analyzed regions are near critical, they are bound to exhibit power-law decaying covariance-matrix spectra. Thus, we conjecture that critical behavior creates the broad range of covariance scales, needed for neural networks to support optimal input representations with power-law decaying eigenvalues. More research is needed to confirm this conjecture and put it on firmer ground. 
As a first step to further understand the relationship between internal recurrent dynamics and the representation of external inputs in a well-controlled example, we have recently designed and analyzed a simple machine-learning model based on the paradigm of reservoir computing [71]. This consists in a recurrent network of coupled units/neurons, which receive (shared) external inputs, giving raise to reverberating activity within the network or "reservoir". Contrarily to other machine-learning paradigms, the internal synaptic weights remain fixed during the training process: only a smaller subset of links connecting to a set of readout nodes change during training, which makes reservoircomputing a versatile tool for diverse computational tasks [72]. Inspired by the experiments of Stringer et al.,the task we chose was the classification of a set of images, with the main goal of scrutinizing for which internal dynamical regime the network was able to achieve the best possible performance. We refer the interested reader to [71] for further details. For our purposes here, it suffices to recall that the best performance is obtained when the tunable control parameters are set in such a way that the overall dynamical state is very close to, but below, the edge of instability. Moreover, at such an operational regime, the spectrum of the covariance matrix obeys the mathematical requirement for optimal representations, i.e., $\mu \gtrsim 1$, as observed for actual neural networks [8].

We find quite suggestive that such a relatively-simple artificial neural network becomes optimal in a regime that shares crucial statistical properties of the covariances with actual neural networks in the mouse brain. Thus, we believe that this machine-learning model may constitute a well-controlled starting point to further investigate the interplay between internal dynamics and external shared inputs in more realistic models of brain activity and to scrutinize optimal input representations. In any case, this seems to confirm that scale-invariant covariance spectra, as a naturally emerging property of critical systems, constitute an excellent breeding ground for optimal information storage.

\subsection{Avalanche criticality versus edge-of-instability}

As already discussed, we have found strong empirical evidence of critical behavior in the sense of vicinity to the edge of instability across brain regions. This type of behavior - called traditionally "edge of chaos" or simply "type-II criticality" in [39] — has long been (since the pioneering works of Langton and others $[73,74]$ ) theoretically conjectured to be crucial for information processing in natural and artificial neural networks. In this case, edge-of-instability systems are characterized by the presence of many modes that are close to become unstable, and thus there is a large repertoire of possible dynamical states that can be excited, opening many possible channels to information processing and transmission in real time [74, 75, 13].

However, in the analyzed neural recordings there are not large fluctuations in the overall level of global activity across time, in agreement with what observed for the motor cortex of awake macaque monkeys in [39] and with the expectation for "asynchronous states" in balanced networks [65, 66]. It is worth to note, however, that in some other empirical observations diverse levels of temporal variability in collective firing rates have been also reported with the possibility of bursts and avalanching behavior (see, e.g., [14, 40, 42]). Actually, as stated in the Introduction, much attention has been paid to "avalanche criticality" (referred as "type-I criticality" in [39]), which is associated with critically balanced networks in which there is an overall-activity mode lying at the edge of instability, thus generating scale-free avalanches of activity, while the rest of modes are usually assumed to be stable. Let us underline that scale-free avalanches can also occur at the edge of synchronization phase transitions $[24,35,76]$.

It should be emphasized that both types of criticality are not mutually exclusive as, in principle, it is possible to have a whole set of eigenvalues, including the overall-activity one, at the edge of instability. Similarly, the system could be at the edge of becoming collectively oscillatory (as recently proposed for large-scale brain networks in e.g. $[24,35])$ if the leading eigenvalue crosses the instability threshold with a non-vanishing imaginary part and at the same time have many other modes near the edge of instability as found here. As an illustrative example, let us mention that a computational model of spiking neurons, including both "asynchronous states" and a synchronization phase transition characterized by scale-free avalanches, has been recently proposed and analyzed [35]. Thus, it seems 
perfectly possible to construct computational models exhibiting both types of criticality in which the system can shift between different regimes depending on its needs. For instance, edge-of-chaos criticality supports local computational tasks, while emerging synchronous oscillations are useful for information transfer and reliable communication with distant areas [77]. In our view, it is likely that actual neuronal networks in the brain exploit these two complementary ways of being critical to achieve diverse functional advantages for different tasks, possibly by shifting their dynamical regimes in response to stimuli (see, e.g., [39]).

\section{Conclusions}

We have developed a unified theoretical framework which relies on recently proposed breakingthrough approaches, but that also extends and combines them in a synergistic way, allowing us to analyze state-of-the-art recordings of the activity of many neurons across brain regions in the mouse. We find that all regions exhibit scale invariance and that all of them operate, to a greater or lesser extent, in a critical regime at the edge of instability. Moreover, we have shown that the resulting scaling in covariances can have very important functional applications for information storage, as it facilitates the generation of optimal input representations. It is our hope that the present work stimulates further research on the remaining open questions and helps advance towards a more comprehensive understanding of the overall dynamics of brain networks and their emerging computational properties , as well as to disentangle universal and non-universal aspects across regions and behavioral states.

\section{Materials and Methods}

\subsection{Datasets}

In the experimental setup of Steinmetz et al., mice were exposed to visual stimuli of varying contrast that could appear on the left side, right side, both sides or neither side of a screen. Mice then earned a water reward by turning or holding still a wheel, depending on the type of stimuli. During task performance, two or three Neuropixels probes were inserted at a time in the left hemisphere, allowing for high-resolution simultaneous-recordings of hundreds of neurons in several regions during each recording session [52]. Thus, for each session the dataset provides the time-stamps and amplitudes of all measured spikes within various brain regions for a total recorded time $T$. In our analysis, we first collected all spikes belonging to a given region, provided it contained more than 256 simultaneously recorded neurons. Then, an optimal time bin $\Delta t$ was inferred for each area on the basis of the InterSpike-Interval distribution of its neurons (see Methods: "Time-scale determination" in this section). This allowed us to convert the spike time-stamps of each neuron into discrete trains of spikes, which were later used to infer pair-wise correlations among neurons and, ultimately, perform RG analyses.

On the other hand, in the work of Stringer et al. mice were presented a sequence of 2800 images, each remaining for $0.5 \mathrm{~s}$ and intersperse with gray-screen inter-stimuli intervals lasting a random time between $0.3 \mathrm{~s}$ and $1.1 \mathrm{~s}[8]$. The resulting task-related activity is thus effectively binned into $\Delta t \approx 0.5 \mathrm{~s}$ time intervals (a time resolution that we keep in our analyses) and two repetitions of the same input-presentation sequence were performed to allow for cv-PCA analyses. Experiments were then repeated with 3 different mice and using different dimensions of the input ensemble. To construct d-dimensional stimuli, the natural image database was projected onto a set of basis functions that enforced the required dimensionality [8]. In our analyses we show results for natural, high-dimensional images as well as 4 -d, low-dimensional images. 


\subsection{Phenomenological renormalization-group (RG) approach}

Let us briefly outline the phenomenological RG approach introduced in [50] and [51]. Given a set of $N$ neurons, the empirically-determined activity of the $i$-th neuron at a given time $t_{j}$ is denoted by $\sigma_{i}\left(t_{j}\right)$, where $j \in(1, T)$ labels a discrete number of non-overlapping time bins. Determining the most meaningful size of such time bins is an important technical aspect (see next section); here, we just assume that such an optimal time discretization is given.

As discussed in the main text, a criterion of maximal pairwise correlation is employed to block together neurons at each step $k$ of the RG procedure. In particular, one considers the Pearson's correlation matrix whose elements are given by:

$$
C_{i j}^{(k)}=\frac{\left\langle\delta x_{i}^{(k)} \delta x_{j}^{(k)}\right\rangle}{\sqrt{\left\langle\left(\delta x_{i}^{(k)}\right)^{2}\right\rangle\left\langle\left(\delta x_{j}^{(k)}\right)^{2}\right\rangle}}
$$

where $\delta x_{i}^{(k)}=x_{i}^{(k)}-\left\langle x_{i}^{(k)}\right\rangle$ and $x_{i}^{(k)}$ is the activity of the block-neuron $i$ at step $k$ of the coarse-graining (we identify $x_{i}^{(0)} \equiv \sigma_{i}$ as the activity of neuron $i$ before coarse-graining), while averages are computed across the available discrete time steps.

At the beginning of each RG step, the two most correlated neurons, $i$, and $j_{* i}$, are selected and their activities are added into a new coarse-grained variable:

$$
x_{i}^{(k+1)}=z_{i}^{(k)}\left(x_{i}^{(k)}+x_{j * i}^{(k)}\right) .
$$

where the normalization factor $z_{i}^{k}$ is chosen in such a way that the average non-zero activity of the new variables $x_{i}^{k+1}$ is equal to one. Notice that, owing to such a normalization criterion, activity values are not constrained to fulfill $x_{i}^{k}(t)<1$. Then one proceeds with the second most-correlated pair of neurons and so on, until a set of $N_{k}$ coarse-grained "block neurons", each containing the summed activity of $K=2^{k}$ original neurons, has been constructed.

Iterating this procedure, after $k$ steps there remain only $N_{k}=N / 2^{k}$ coarse-grained variables or "block-neurons", $\left\{x_{i}^{k}\right\}_{i=1,2 \ldots N_{k}}$, each recapitulating the activity of $K=2^{k}$ individual neurons. To figure out whether a fixed point of the RG flow exists, one can study the evolution of the probability density function for the activity of the coarse-grained variables, $P_{k}(x)$. Following [50], we separated $P_{k}(x)$ for block-neurons of size $2^{k}$ in two components: the probability of being silent, $S_{k} \equiv P_{k}(x=0)$, and the probability $Q_{k}(x)$ of having non-zero activity $x$ :

$$
P_{k}(x)=S_{k}+Q_{k}(x) .
$$

Trivially, if the original neurons were statistically independent, one would expect (as a direct consequence of the central limit theorem) to drive the activity distribution $Q_{k}$ towards a Gaussian fixedpoint of the RG flow [78]. As pointed out in [50], the RG convergence to a non-Gaussian fixed-point (i.e. invariance of the distribution across RG steps) reveals a non-trivial structure in the data.

Another quantity of interest is the variance of the activity distributions as a function of the size of the block neurons $K$ :

$$
M_{2}(K)=\frac{1}{N_{k}} \sum_{i=1}^{N_{k}}\left[\left\langle\left(\sigma_{i}^{(k)}\right)^{2}\right\rangle-\left\langle\left(\sigma_{i}^{(k)}\right)\right\rangle^{2}\right]
$$

where $\sigma_{i}^{(k)}$ is the summed activity of the original variables inside the cluster. Notice that, for totally independent variables, one would expect the variance to grow linearly in $K$ (i.e., $M_{2}(K) \propto K$ ), whereas if variables were perfectly correlated $M_{2}(K) \propto K^{2}$. Non-trivial scaling is therefore characterized by

$$
M_{2}(K) \propto K^{\alpha}
$$


with a certain intermediate value of the exponent $1<\alpha<2$.

On the other hand,

$$
F_{k}=-\log \left(S_{k}\right)
$$

defines a sort of "free-energy" for the coarse-grained variables at the $k$-th RG step [50]. As more and more of the initial variables $\sigma_{i}$ are grouped into cluster variables $x_{i}^{(k)}$, one would expect that the probability of having "silent" block-neurons (i.e., the probability that all neurons inside a cluster are silent) decreases exponentially with the size $K$ of the clusters, leading to:

$$
F(K) \propto K^{\beta}
$$

where $\beta=1$ for initially independent variables.

One can also wonder whether there is some type of self-similarity in the dynamics at coarse-grained scales. Given that, commonly, fluctuations on larger spatial scales relax with a slower characteristic time scale, we should expect the time-lagged Pearson's correlation function (or simply autocorrelation function) of the coarse-grained variables to decay more slowly as we average over more neurons. In particular, for step $k$ of the RG flow, one has:

$$
C^{(k)}(t)=\frac{1}{N_{k}} \sum_{i=1}^{N_{k}} \frac{\left\langle x_{i}^{(k)}\left(t_{0}\right) x_{i}^{(k)}\left(t_{0}+t\right)\right\rangle-\left\langle x_{i}^{(k)}\right\rangle^{2}}{\left\langle\left(x_{i}^{(k)}\right)^{2}\right\rangle-\left\langle x_{i}^{(k)}\right\rangle^{2}}
$$

Assuming that correlations decay exponentially in time with a characteristic time scale $\tau_{c}^{(k)}$ (i.e., $\left.C^{(k)}(t)=e^{-t / \tau_{c}^{(k)}}\right)$ at each coarse-graining level, dynamical scaling implies that the average correlation function collapses into a single curve when time is re-scaled by the characteristic time scale:

$$
C^{(k)}(t)=C\left[t / \tau_{c}^{(k)}\right]
$$

and that this time scale is in turn a power-law function of the number of neurons inside the clusters:

$$
\tau_{c}(K) \propto K^{z}
$$

where $z$ is the dynamical scaling exponent. Finally, as argued in [50], if correlations are self-similar then we should see this by looking inside the clusters of size $K$. In particular, the eigenvalues of the covariance matrix (i.e., the propagator, which is scale-invariant at the fixed-point of the RG in systems with translational invariance [50]) must obey a power-law dependence on the fractional rank:

$$
\lambda=B\left(\frac{K}{\operatorname{rank}}\right)^{\mu}
$$

where $B$ is a constant and $\mu$ a decay exponent.

To compute an error on the estimated exponents we follow the approach in [51]: for each region, we reproduce the RG analysis over random quarters (batches) of the data, with points remaining in order within these intervals to respect temporal correlations. We then estimate for each batch the scaling exponents as the slope of the best fit in a log-log scale [79]. Our final estimation and error for any scaling exponent within a region is then computed as the mean and standard deviation across all batches, respectively (see Table S1 in SI for values of all the exponents measured over background activity).

\subsection{Time-scale determination}

In order to determine pairwise correlations from empirical neural activity data, it is necessary to discretize the time into bins of certain length $\Delta t$. In our case, to obtain a spike train vector from a list of time stamps $t_{j}^{*}$ at which a neuron spikes, a common value $\Delta t$ is chosen for all units. In this 


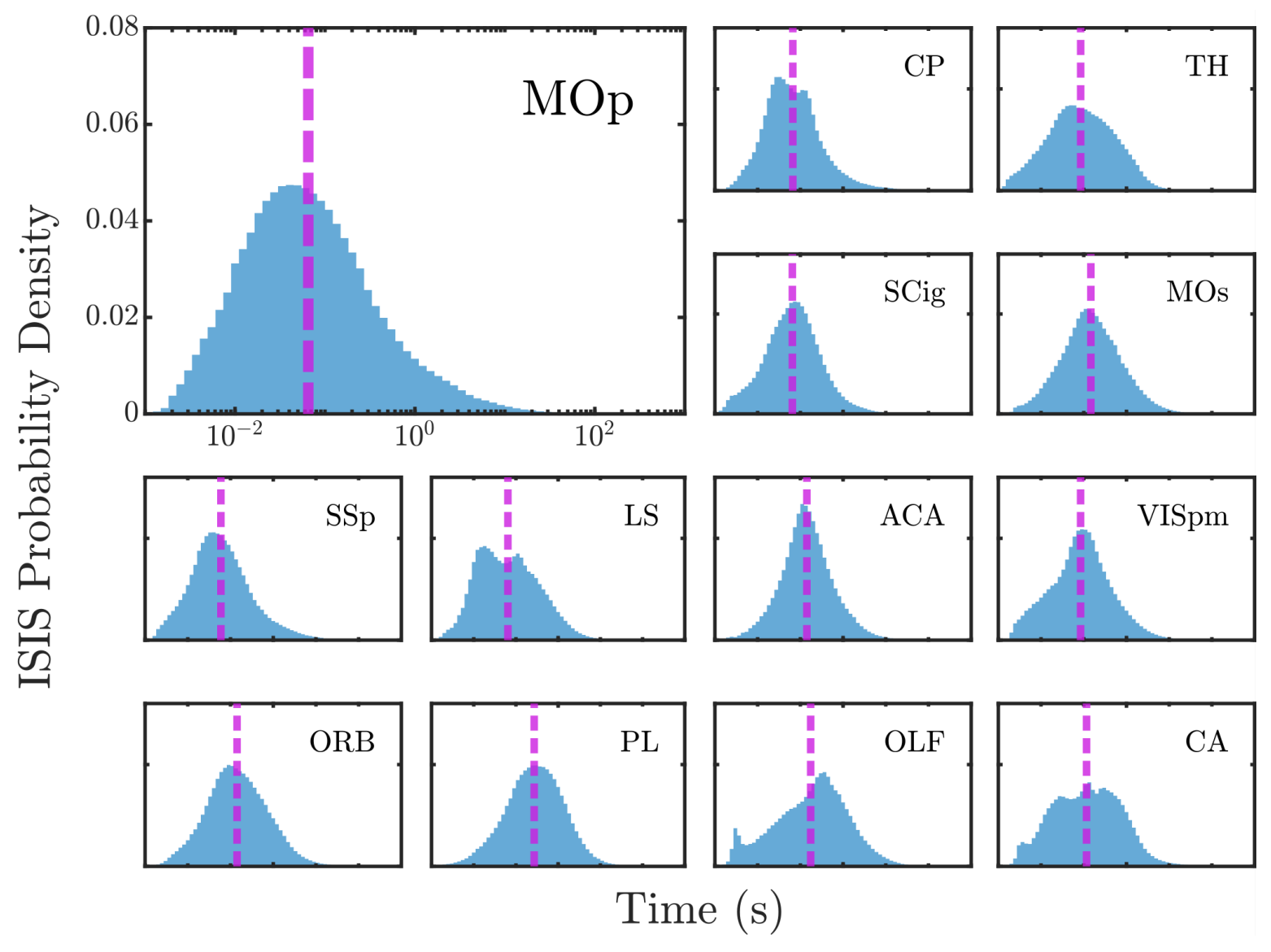

Figure 6: Individual-neuron inter-spike interval distributions for 13 different regions of the mouse brain as determined using all recorded neurons in each population (for each of them, there is a large neuron-to-neuron variability as shown in Fig.S1). The characteristic or "optimal" time bin is defined as the geometric mean of the ISI distribution (vertical dashed lines).

way, neurons spiking at times $t_{j}$ and $t_{j}+\delta t$ sufficiently close share a bin $\Delta t_{j}$ with non-zero activity. The number of bins for a neuron can be therefore chosen by $T_{i}=\frac{\max \left(t_{j}^{*}\right)}{\Delta t}$, and more generally we will set $T=\max \left(T_{i}\right)$ so that all neurons have the same amount of bins in their spike trains.

Choosing the time bin that best transforms the spike times into discrete trains of spikes is an open problem in neuroscience for which several solutions — such as the use of random bins [80], methods that find the best bin size by minimizing a certain cost function $[81,82,83,32,84]$ and bin-less approaches $[85,86]$ - have been proposed. The problem becomes even more prominent when working with the simultaneously-recorded activity of a large number of cells as, typically, one finds neurons operating at broadly different time scales within the same area (see SI Fig.S2), as well as a hierarchy of timescales across regions $[87,88]$.

To perform the RG analyses, we first compute the distribution of inter-spike intervals (ISI) of individual neurons within a particular brain region (Fig.6). Because we are interested in a measure of the "typical" time-scale at which neurons in a population operate, and given also the large neuronto-neuron variability, we define the optimal time bin $\Delta t$ as the geometric mean of all ISIs from neurons in the population (which, as illustrated in Fig.6, is a very good estimator of the population characteristic time scale); this geometric-mean value is computed for each region and subsequently used in the corresponding RG analysis (see Table S1 in the SI for a summary of all areas with their corresponding selected time bin). 


\subsection{Noise and signal covariance}

Along this work we use two different types of covariance matrices, as derived for "signal" and "noise" pairwise covariances, respectively [57, 58].

The signal covariance, or simply "covariance" here, measures the pair-wise correlation in the average response of two neurons to a set of stimuli. $c_{i j}^{s}$ is

$$
c_{i j}^{s}=\left\langle\left(x_{i}-\left\langle x_{i}\right\rangle\right)\left(x_{j}-\left\langle x_{j}\right\rangle\right\rangle\right.
$$

where averages are taken along the recorded time $T$ (i.e. as the normalized sum over the available discrete time bins). The elements in Eq.(14) define the covariance matrix whose spectrum is computed in the RG analyses as well as in the studies of input representations.

On the other hand, the spike-count or noise covariance $c_{i j}^{n}$ is defined as:

$$
c_{i j}^{n}=\frac{1}{T}\left(\left\langle n_{i} n_{j}\right\rangle-\left\langle n_{i}\right\rangle\left\langle n_{j}\right\rangle\right)
$$

where $n_{i}$ simply denotes the number of spikes coming from neuron $i$ during a recorded time interval $T$, and averages are taken over trials of the same experiment. It can be easily shown that, for large observation times $T_{0} \longrightarrow \infty$, this can be written as the time integral of the time-lagged (signal) covariance $c_{i j}(\tau)[39]$ :

$$
c_{i j}^{n}=\lim _{T_{0} \longrightarrow \infty} \int_{-T_{0}}^{T_{0}} \frac{T_{0}-\tau}{T_{0}} c_{i j}^{s}(\tau) d \tau .
$$

Loosely speaking, this integral over all time differences removes time-dependent effects from the covariances and puts the emphasis onto signal-independent pairwise heterogeneities.

Since for each of the analyzed brain regions there is only one very long recording of resting-state activity, in order to compute averages in Eq.(15), we split the full time-series into a set of around 50 intervals, each containing at least $T_{0}=15$ seconds of recorded activity.

\section{Acknowledgements}

We acknowledge the Spanish Ministry and Agencia Estatal de investigación (AEI) through Project of I+D+i Ref. PID2020-113681GB-I00, financed by MICIN/AEI/10.13039/501100011033 and FEDER "A way to make Europe", as well as the Consejería de Conocimiento, Investigación Universidad, Junta de Andalucía and European Regional Development Fund, Project references A-FQM-175-UGR18 and P20-00173 for financial support. We also thank V. Buendía, P.Villegas, R. Corral, J. Pretel, P. Moretti, M. Ibañez, P. Garrido, and M. Marsili, for valuable discussions and/or suggestions on earlier versions of the manuscript.

\section{References}

[1] William R Softky and Christof Koch. The highly irregular firing of cortical cells is inconsistent with temporal integration of random epsps. J. Neurosci., 13(1):334-350, 1993.

[2] Amos Arieli, Alexander Sterkin, Amiram Grinvald, and AD Aertsen. Dynamics of ongoing activity: explanation of the large variability in evoked cortical responses. Science, 273(5283):1868, 1996.

[3] Marcus E Raichle. The restless brain. Brain connectivity, 1(1):3-12, 2011.

[4] Jessica S Damoiseaux, SARB Rombouts, Frederik Barkhof, Philip Scheltens, Cornelis J Stam, Stephen M Smith, and Christian F Beckmann. Consistent resting-state networks across healthy subjects. Proc. Natl. Acad. Sci. U.S.A., 103(37):13848-13853, 2006. 
[5] Gustavo Deco, Viktor K Jirsa, and Anthony R McIntosh. Resting brains never rest: computational insights into potential cognitive architectures. Trends in Neurosci., 36(5):268-274, 2013.

[6] Gustavo Deco, Viktor K Jirsa, Peter A Robinson, Michael Breakspear, and Karl Friston. The dynamic brain: from spiking neurons to neural masses and cortical fields. PLoS Comp. Biol., 4(8):e1000092, 2008.

[7] Carsen Stringer, Marius Pachitariu, Nicholas Steinmetz, Charu Bai Reddy, Matteo Carandini, and Kenneth D Harris. Spontaneous behaviors drive multidimensional, brainwide activity. Science, 364(6437), 2019.

[8] Carsen Stringer, Marius Pachitariu, Nicholas Steinmetz, Matteo Carandini, and Kenneth D Harris. High-dimensional geometry of population responses in visual cortex. Nature, 571(7765):361$365,2019$.

[9] Christoph Stosiek, Olga Garaschuk, Knut Holthoff, and Arthur Konnerth. In vivo two-photon calcium imaging of neuronal networks. Proceedings of the National Academy of Sciences, 100(12):7319-7324, 2003.

[10] Ashley L Juavinett, George Bekheet, and Anne K Churchland. Chronically implanted neuropixels probes enable high-yield recordings in freely moving mice. Elife, 8:e47188, 2019.

[11] T. Mora and W. Bialek. Are biological systems poised at criticality? J. Stat. Phys., 144(2):268$302,2011$.

[12] William Bialek. Biophysics: searching for principles. Princeton University Press, 2012.

[13] Miguel A. Muñoz. Colloquium: Criticality and dynamical scaling in living systems. Rev. Mod. Phys., 90(3):031001, July 2018.

[14] John M Beggs and Dietmar Plenz. Neuronal avalanches in neocortical circuits. J.Neurosci., 23(35):11167-11177, 2003.

[15] Dante R Chialvo. Emergent complex neural dynamics. Nature physics, 6(10):744-750, 2010.

[16] Woodrow L Shew and Dietmar Plenz. The functional benefits of criticality in the cortex. The Neuroscientist, 19(1):88-100, 2013.

[17] Shan Yu, Tiago L Ribeiro, Christian Meisel, Samantha Chou, Andrew Mitz, Richard Saunders, and Dietmar Plenz. Maintained avalanche dynamics during task-induced changes of neuronal activity in nonhuman primates. Elife, 6:e27119, 2017.

[18] Luca Cocchi, Leonardo L Gollo, Andrew Zalesky, and Michael Breakspear. Criticality in the brain: A synthesis of neurobiology, models and cognition. Progress in Neurobiology, 2017.

[19] Paolo Massobrio, Lucilla de Arcangelis, Valentina Pasquale, Henrik J Jensen, and Dietmar Plenz. Criticality as a signature of healthy neural systems. Frontiers in systems neuroscience, 9:22, 2015.

[20] Viola Priesemann, Michael Wibral, Mario Valderrama, Robert Pröpper, Michel Le Van Quyen, Theo Geisel, Jochen Triesch, Danko Nikolić, and Matthias HJ Munk. Spike avalanches in vivo suggest a driven, slightly subcritical brain state. Frontiers in systems neuroscience, 8:108, 2014.

[21] Jens Wilting and Viola Priesemann. 25 years of criticality in neuroscience - established results, open controversies, novel concepts. Current opinion in neurobiology, 58:105-111, 2019.

[22] Dietmar Plenz, Tiago L Ribeiro, Stephanie R Miller, Patrick A Kells, Ali Vakili, and Elliott L Capek. Self-organized criticality in the brain. arXiv preprint arXiv:2102.09124, 2021. 
[23] Anna Levina, J Michael Herrmann, and Theo Geisel. Dynamical synapses causing self-organized criticality in neural networks. Nature physics, 3(12):857-860, 2007.

[24] Serena di Santo, Pablo Villegas, Raffaella Burioni, and Miguel A Muñoz. Landau-ginzburg theory of cortex dynamics: Scale-free avalanches emerge at the edge of synchronization. Proceedings of the National Academy of Sciences, 115(7):E1356-E1365, 2018.

[25] Matteo Martinello, Jorge Hidalgo, Amos Maritan, Serena Di Santo, Dietmar Plenz, and Miguel A Muñoz. Neutral theory and scale-free neural dynamics. Physical Review X, 7(4):041071, 2017.

[26] Victor Buendía, Pablo Villegas, Raffaella Burioni, and Miguel A Muñoz. Hybrid-type synchronization transitions: Where incipient oscillations, scale-free avalanches, and bistability live together. Physical Review Research, 3(2):023224, 2021.

[27] Osame Kinouchi and Mauro Copelli. Optimal dynamical range of excitable networks at criticality. Nature physics, 2(5):348-351, 2006.

[28] Mark EJ Newman. Power laws, pareto distributions and zipf's law. Contemporary physics, 46(5):323-351, 2005.

[29] James J Binney, Nigel J Dowrick, Andrew J Fisher, and Mark EJ Newman. The theory of critical phenomena: an introduction to the renormalization group. Oxford University Press, 1992.

[30] Jonathan Touboul and Alain Destexhe. Power-law statistics and universal scaling in the absence of criticality. Phys. Rev. E, 95(1):012413, 2017.

[31] John M Beggs and Nicholas Timme. Being critical of criticality in the brain. Frontiers in physiology, 3:163, 2012.

[32] Ryan John Cubero, Junghyo Jo, Matteo Marsili, Yasser Roudi, and Juyong Song. Statistical criticality arises in most informative representations. Journal of Statistical Mechanics: Theory and Experiment, 2019(6):063402, June 2019. Publisher: IOP Publishing.

[33] Paolo Moretti and Miguel A Muñoz. Griffiths phases and the stretching of criticality in brain networks. Nature communications, 4(1):1-10, 2013.

[34] Hongdian Yang, Woodrow L Shew, Rajarshi Roy, and Dietmar Plenz. Maximal variability of phase synchrony in cortical networks with neuronal avalanches. J. Neurosci., 32(3):1061-1072, 2012.

[35] Junhao Liang, Tianshou Zhou, and Changsong Zhou. Hopf bifurcation in mean field explains critical avalanches in excitation-inhibition balanced neuronal networks: A mechanism for multiscale variability. Front. Systems Neurosci., 14:87, 2020.

[36] Pablo Villegas, Paolo Moretti, and Miguel A Munoz. Frustrated hierarchical synchronization and emergent complexity in the human connectome network. Scientific reports, 4(1):1-7, 2014.

[37] Alistair Steyn-Ross and Moira Steyn-Ross. Modeling phase transitions in the brain, volume 509. Springer, 2010.

[38] Guillermo Solovey, Leandro M Alonso, Toru Yanagawa, Naotaka Fujii, Marcelo O Magnasco, Guillermo A Cecchi, and Alex Proekt. Loss of consciousness is associated with stabilization of cortical activity. Journal of Neuroscience, 35(30):10866-10877, 2015.

[39] David Dahmen, Sonja Grün, Markus Diesmann, and Moritz Helias. Second type of criticality in the brain uncovers rich multiple-neuron dynamics. Proceedings of the National Academy of Sciences, 116(26):13051-13060, June 2019. 
[40] Thomas Petermann, Tara C Thiagarajan, Mikhail A Lebedev, Miguel AL Nicolelis, Dante R Chialvo, and Dietmar Plenz. Spontaneous cortical activity in awake monkeys composed of neuronal avalanches. Proc. Natl. Acad. Sci. USA, 106(37):15921-15926, 2009.

[41] Zhengyu Ma, Gina G Turrigiano, Ralf Wessel, and Keith B Hengen. Cortical circuit dynamics are homeostatically tuned to criticality in vivo. Neuron, 104(4):655-664, 2019.

[42] Antonio J Fontenele, Nivaldo AP de Vasconcelos, Thaís Feliciano, Leandro AA Aguiar, Carina Soares-Cunha, Bárbara Coimbra, Leonardo Dalla Porta, Sidarta Ribeiro, Ana João Rodrigues, Nuno Sousa, et al. Criticality between cortical states. Physical Review Letters, 122(20):208101, 2019.

[43] Simon-Shlomo Poil, Richard Hardstone, Huibert D Mansvelder, and Klaus Linkenkaer-Hansen. Critical-state dynamics of avalanches and oscillations jointly emerge from balanced excitation/inhibition in neuronal networks. J. Neurosci., 32(29):9817-9823, 2012.

[44] J Matias Palva, Alexander Zhigalov, Jonni Hirvonen, Onerva Korhonen, Klaus LinkenkaerHansen, and Satu Palva. Neuronal long-range temporal correlations and avalanche dynamics are correlated with behavioral scaling laws. Proc. Nat. Acad. Sci. USA, 110(9):3585-3590, 2013.

[45] Gustavo Deco and Viktor K Jirsa. Ongoing cortical activity at rest: Criticality, multistability, and ghost attractors. J. Neurosci., 32(10):3366-3375, 2012.

[46] Joana Cabral, Morten L Kringelbach, and Gustavo Deco. Functional connectivity dynamically evolves on multiple time scales over a static structural connectome: Models and mechanisms. NeuroImage, 160:84-96, 2017.

[47] Enzo Tagliazucchi, Pablo Balenzuela, Daniel Fraiman, and Dante R Chialvo. Criticality in largescale brain fmri dynamics unveiled by a novel point process analysis. Frontiers in physiology, 3:15, 2012.

[48] Rong Wang, Pan Lin, Mianxin Liu, Ying Wu, Tao Zhou, and Changsong Zhou. Hierarchical connectome modes and critical state jointly maximize human brain functional diversity. Physical review letters, 123(3):038301, 2019.

[49] Erik D Fagerholm, W Matthew C Foulkes, Yasir Gallero-Salas, Fritjof Helmchen, Karl J Friston, Robert Leech, and Rosalyn J Moran. Neural systems under change of scale. Frontiers in Computational Neuroscience, 15:33, 2021.

[50] Leenoy Meshulam, Jeffrey L. Gauthier, Carlos D. Brody, David W. Tank, and William Bialek. Coarse Graining, Fixed Points, and Scaling in a Large Population of Neurons. Physical Review Letters, 123(17):178103, October 2019. Publisher: American Physical Society.

[51] Leenoy Meshulam, Jeffrey L Gauthier, Carlos D Brody, David W Tank, and William Bialek. Coarse-graining and hints of scaling in a population of $1000+$ neurons. arXiv preprint arXiv:1812.11904, 2018.

[52] Nicholas A. Steinmetz, Peter Zatka-Haas, Matteo Carandini, and Kenneth D. Harris. Distributed coding of choice, action and engagement across the mouse brain. Nature, 576(7786):266-273, December 2019. Number: 7786 Publisher: Nature Publishing Group.

[53] Mehran Kardar. Statistical physics of fields. Cambridge University Press, 2007.

[54] Serena Bradde and William Bialek. PCA Meets RG. Journal of Statistical Physics, 167(3):462475, May 2017. 
[55] Mia C Morrell, Audrey J Sederberg, and Ilya Nemenman. Latent dynamical variables produce signatures of spatiotemporal criticality in large biological systems. Physical review letters, 126(11):118302, 2021.

[56] Giorgio Nicoletti, Samir Suweis, and Amos Maritan. Scaling and criticality in a phenomenological renormalization group. Physical Review Research, 2(2):023144, May 2020. Publisher: American Physical Society.

[57] Marlene R. Cohen and Adam Kohn. Measuring and interpreting neuronal correlations. Nature Neuroscience, 14(7):811-819, July 2011. Number: 7 Publisher: Nature Publishing Group.

[58] $\mathrm{Yu} \mathrm{Hu}$ and Haim Sompolinsky. The spectrum of covariance matrices of randomly connected recurrent neuronal networks. bioRxiv, 2020.

[59] Joël Bun, Jean-Philippe Bouchaud, and Marc Potters. Cleaning large correlation matrices: tools from random matrix theory. Physics Reports, 666:1-109, 2017.

[60] Michael E Rule, Timothy O'Leary, and Christopher D Harvey. Causes and consequences of representational drift. Current opinion in neurobiology, 58:141-147, 2019.

[61] Jonathon Shlens. A tutorial on principal component analysis. arXiv preprint arXiv:1404.1100, 2014.

[62] Peiran Gao and Surya Ganguli. On simplicity and complexity in the brave new world of large-scale neuroscience. Current opinion in neurobiology, 32:148-155, 2015.

[63] Daniel J Amit and Victor Martín-Mayor. Field theory, the renormalization group, and critical phenomena: graphs to computers. Springer, 2005.

[64] James Trousdale, Yu Hu, Eric Shea-Brown, and Krešimir Josić. Impact of Network Structure and Cellular Response on Spike Time Correlations. PLOS Computational Biology, 8(3):e1002408, March 2012. Publisher: Public Library of Science.

[65] Carl Van Vreeswijk and Haim Sompolinsky. Chaos in neuronal networks with balanced excitatory and inhibitory activity. Science, 274(5293):1724-1726, 1996.

[66] Alfonso Renart, Jaime De La Rocha, Peter Bartho, Liad Hollender, Néstor Parga, Alex Reyes, and Kenneth D Harris. The asynchronous state in cortical circuits. Science, 327(5965):587-590, 2010.

[67] Giordano De Marzo, Andrea Gabrielli, Andrea Zaccaria, and Luciano Pietronero. Dynamical approach to zipf's law. Physical Review Research, 3(1):013084, 2021.

[68] Laurence Aitchison, Nicola Corradi, and Peter E Latham. Zipf's law arises naturally when there are underlying, unobserved variables. PLoS computational biology, 12(12):e1005110, 2016.

[69] David J Schwab, Ilya Nemenman, and Pankaj Mehta. Zipf's law and criticality in multivariate data without fine-tuning. Physical Review Letters, 113(6):068102, 2014.

[70] Matteo Marsili. The peculiar statistical mechanics of optimal learning machines. Journal of Statistical Mechanics: Theory and Experiment, 2019(10):103401, 2019.

[71] Guillermo B Morales and Miguel A Muñoz. Optimal input representation in neural systems at the edge of chaos. Biology, 10(8):702, 2021.

[72] Gouhei Tanaka, Toshiyuki Yamane, Jean Benoit Héroux, Ryosho Nakane, Naoki Kanazawa, Seiji Takeda, Hidetoshi Numata, Daiju Nakano, and Akira Hirose. Recent advances in physical reservoir computing: A review. Neural Networks, 115:100-123, 2019. 
[73] Chris G Langton. Computation at the edge of chaos: Phase transitions and emergent computation. Physica D: Nonlinear Phenomena, 42(1-3):12-37, 1990.

[74] Nils Bertschinger and Thomas Natschläger. Real-time computation at the edge of chaos in recurrent neural networks. Neural computation, 16(7):1413-1436, 2004.

[75] Joschka Boedecker, Oliver Obst, Joseph T Lizier, N Michael Mayer, and Minoru Asada. Information processing in echo state networks at the edge of chaos. Theory in Biosciences, 131(3):205-213, 2012.

[76] Victor Buendía, Pablo Villegas, Raffaella Burioni, and Miguel A. Muñoz. Hybrid-type synchronization transitions: Where incipient oscillations, scale-free avalanches, and bistability live together. Phys. Rev. Research, 3:023224, Jun 2021.

[77] Yuzhen Qin, Tommaso Menara, Danielle S Bassett, and Fabio Pasqualetti. Phase-amplitude coupling in neuronal oscillator networks. Physical Review Research, 3(2):023218, 2021.

[78] Giovanni Jona-Lasinio. Renormalization Group and Probability Theory. Physics Reports, 352(46):439-458, October 2001. arXiv: cond-mat/0009219.

[79] Aaron Clauset, Cosma Rohilla Shalizi, and Mark EJ Newman. Power-law distributions in empirical data. SIAM review, 51(4):661-703, 2009.

[80] Shinichi Tamura, Tomomitsu Miyoshi, Hajime Sawai, and Yuko Mizuno-Matsumoto. Random Bin for Analyzing Neuron Spike Trains. Computational Intelligence and Neuroscience, 2012:e153496, July 2012. Publisher: Hindawi.

[81] Takahiro Omi and Shigeru Shinomoto. Optimizing Time Histograms for Non-Poissonian Spike Trains. Neural Computation, 23(12):3125-3144, December 2011.

[82] Bernhard Schölkopf, John Platt, and Thomas Hofmann. A recipe for optimizing a time-histogram. In Advances in Neural Information Processing Systems 19: Proceedings of the 2006 Conference, pages 1289-1296. MIT Press, 2007. Conference Name: Advances in Neural Information Processing Systems 19: Proceedings of the 2006 Conference.

[83] Ali Ghazizadeh and Frederic Ambroggi. Optimal Binning of Peri-Event Time Histograms Using Akaike Information Criterion. bioRxiv, page 2020.02.06.937367, February 2020. Publisher: Cold Spring Harbor Laboratory Section: New Results.

[84] Ryan John Cubero, Matteo Marsili, and Yasser Roudi. Multiscale relevance and informative encoding in neuronal spike trains. Journal of computational neuroscience, 48(1):85-102, 2020.

[85] Jonathan D. Victor. Binless strategies for estimation of information from neural data. Physical Review E, 66(5):051903, November 2002. Publisher: American Physical Society.

[86] António R. C. Paiva, Il Park, and José C. Príncipe. A comparison of binless spike train measures. Neural Computing and Applications, 19(3):405-419, April 2010.

[87] Stefan J. Kiebel, Jean Daunizeau, and Karl J. Friston. A Hierarchy of Time-Scales and the Brain. PLOS Computational Biology, 4(11):e1000209, November 2008. Publisher: Public Library of Science.

[88] Mehran Spitmaan, Hyojung Seo, Daeyeol Lee, and Alireza Soltani. Multiple timescales of neural dynamics and integration of task-relevant signals across cortex. Proceedings of the National Academy of Sciences, 117(36):22522-22531, September 2020. Publisher: National Academy of Sciences Section: Biological Sciences. 